\title{
Long-term outcome of left ventricular outflow tract after biventricular repair using Damus-Kaye-Stansel anastomosis for interrupted aortic arch and severe aortic stenosis
}

\author{
Shin Takabayashi, MD, Hideaki Kado, MD, Yuichi Shiokawa, MD, Kouji Fukae, MD, \\ and Toshihide Nakano, MD, Fukuoka, Japan
}

I nterrupted aortic arch (IAA) with a ventricular septal defect (VSD) is rare but lethal in association with subaortic stenosis. The primary biventricular repair for this lesion consists of left ventricular outflow tract (LVOT) reconstruction by DamusKaye-Stansel (DKS) anastomosis and intracardiac rerouting through the VSD, along with concomitantly performed right ventricular outflow tract (RVOT) and aortic arch reconstruction (Yasui procedure ${ }^{1}$; Figure 1, A). In this study, we reported long-term follow-ups in 2 patients and estimated the transition of LVOT diameter.

\section{Clinical Summaries}

PATIENT 1. An IAA (type B) was diagnosed in a 36-day-old boy weighing $3.4 \mathrm{~kg}$, as was a perimembranous VSD. The subaortic diameter was $2.6 \mathrm{~mm}$, and the aortic valve (diameter, 3.9 $\mathrm{mm}$ ) was bicuspid. In primary biventricular repair, LVOT reconstruction consisted of intracardiac rerouting from the VSD $(8 \mathrm{~mm})$ to the pulmonary artery by using a Teflon patch and a DKS anastomosis. RVOT reconstruction was performed by the Rastelli procedure with a 14-mm Hancock valved conduit (Medtronic, Inc, Minneapolis, Minn). Aortic arch reconstruction was performed with an 8-mm expanded polytetrafluoroethylene (ePTFE) graft anastomosis between the ascending and descending aorta. When the patient was aged 5 years 9 months, a conduit replacement for a re-RVOT reconstruction with a 16-mm ePTFE graft was required because of weight gain. When he was 7 years old, we performed an additional 12-mm ePTFE graft anastomosis between the left common carotid artery and the descending aorta. The follow-up period is now 16 years, and New York Heart Association is class I.

PATIENT 2. An IAA (type B) was diagnosed in a 42-day-old boy weighing $2.8 \mathrm{~kg}$, as was a perimembranous VSD. The subaortic diameter was $2.8 \mathrm{~mm}$, and the aortic valve (diameter, 3.5 $\mathrm{mm}$ ) was bicuspid. In primary biventricular repair, LVOT reconstruction consisted of intracardiac rerouting from an enlarged VSD $(9 \mathrm{~mm})$ to the pulmonary artery with a polyester patch and DKS

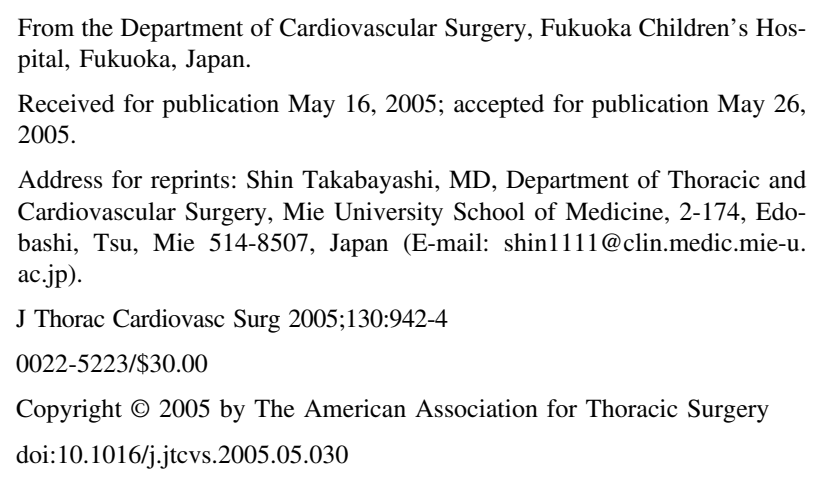

anastomosis. RVOT reconstruction was performed by the Rastelli procedure with a 12-mm Carpenter-Edwards valved conduit (Edwards Lifesciences, Irvine, Calif). Aortic arch reconstruction was performed by direct anastomosis. When the patient was aged 7 years 9 months, a conduit replacement for re-RVOT reconstruction with an 18-mm nonvalved ePTFE graft was necessary. The follow-up period is now 10 years, and the patient is in New York Heart Association class I.

Angiocardiograms were performed after the initial operation 4 times in patient 1 and 5 times in patient 2 . The latest examinations revealed morphologically that there was no LVOT stenosis on a lateral view (Figure 1, B). Furthermore, there was no regurgitation of semilunar valves and no pressure gradient between the left ventricle and the ascending aorta. We also show the transitions of LVOT consisting of each of the parameters (Figure 2, $A$ ) and by each parameter/normal aortic diameters ${ }^{2}$ (Figure 2, $B$ ).

\section{Discussion}

Although operative results of IAA have improved, mortality from IAA with severe subaortic stenosis remains high. ${ }^{3}$ We previously reported primary biventricular repair of IAA and severe aortic stenosis in neonates. ${ }^{1}$ Although good late survival of biventricular repair for patients with aortic hypoplasia and VSD was reported, ${ }^{4}$ detailed outcomes of LVOT over a long-term follow-up have not been previously reported.

In this study, our results showed no progressive LVOT stenosis, as was indicated by linear growth in each site of DKS, VSD, and the subaortic stenosis. Although, in these biventricular repairs, the intracardiac patch insertion between the LVOT and the right ventricle is different from DKS anastomosis in single-ventricle patients, there was no LVOT stenosis over long-term follow-up with the patch used in our patients. It is interesting to note that after another routing, the subaortic stenosis reduced without regression of the original aortic route. No pressure gradient across the reconstructed LVOT and preserved semilunar valve function indicated that our primary biventricular repairs, which added an LVOT route, resulted in excellent outcomes. Although reoperations due to relative stenosis of the artificial graft accompanied patient growth, this possibility would be reduced by the aortic arch and RVOT reconstruction by a modification that used only autologous tissues without conduits. ${ }^{5}$ A limitation of this study is that measurements of angiocardiography in a lateral view to assess the LVOT and the area through which the systemic flow passes are unknown.

In conclusion, LVOT constructed by DKS anastomosis and intracardiac rerouting through the VSD for IAA and VSD with severe aortic stenosis was stable, without progressive stenosis or regurgitation in long-term follow-up. Linear growth of the main route by the systemic flow passages is expected. 

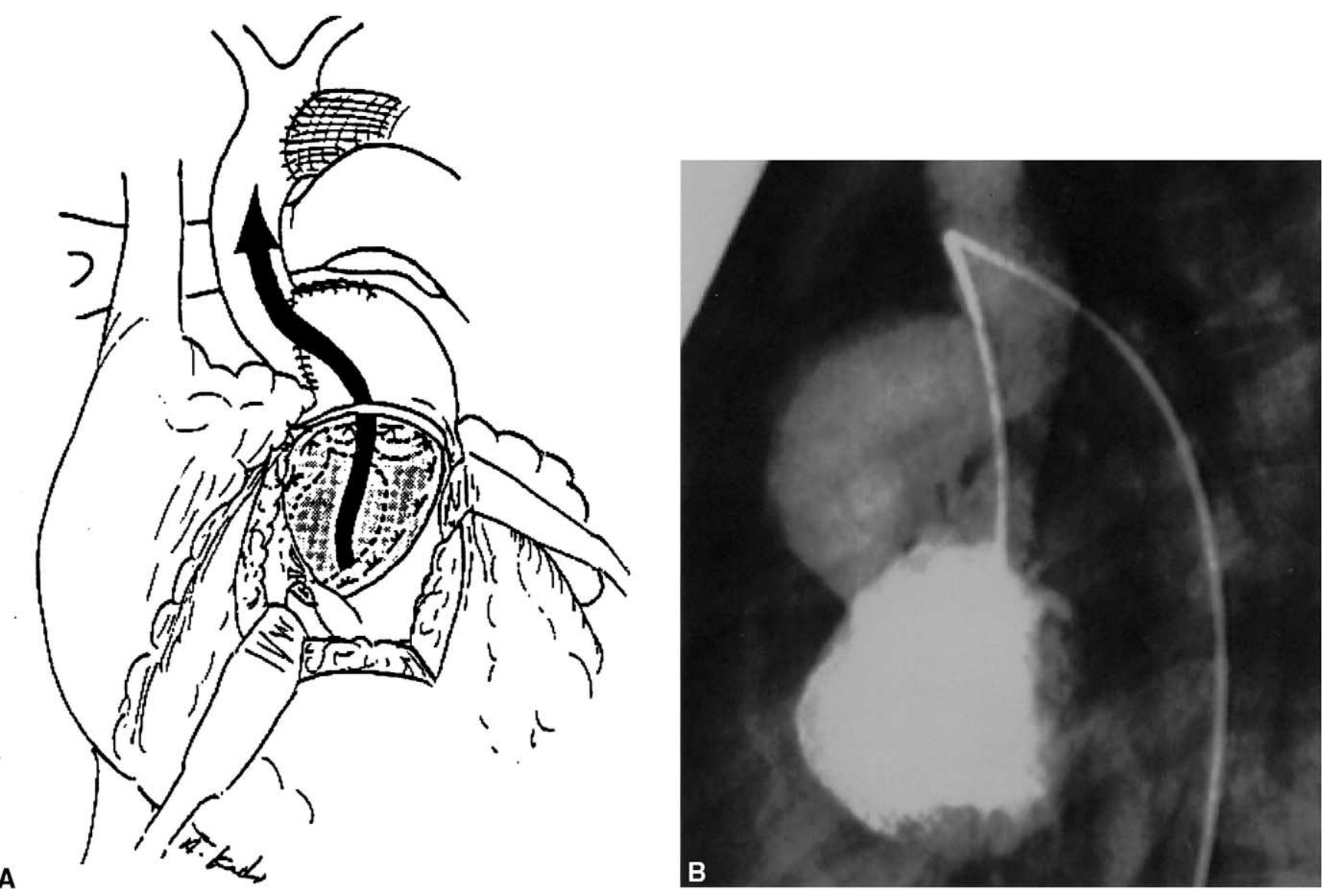

Figure 1. A, Drawing of the Yasui procedure; B, 9.4 years after the biventricular repair.

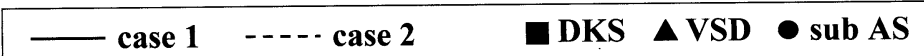
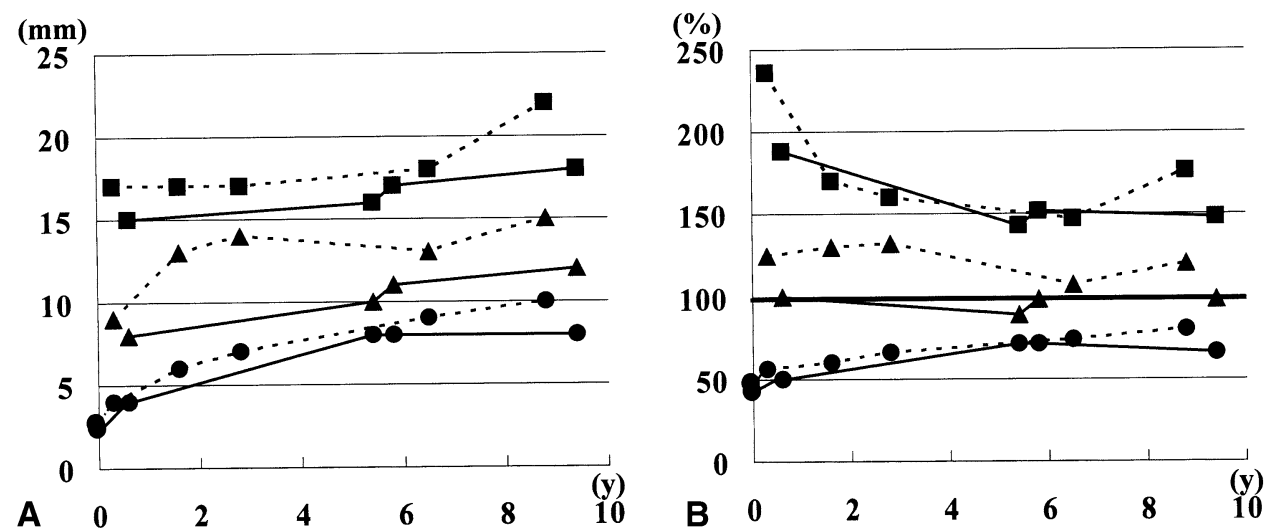

Figure 2. DKS, Damus-Kaye-Stansel anastomosis; VSD, ventricular septal defect; sub AS, subaortic stenosis. 


\section{References}

1. Yasui H, Kado H, Nakano E, Yonenaga K, Mitani A, Tomita Y, et al. Primary repair of interrupted aortic arch and severe aortic stenosis in neonates. J Thorac Cardiovasc Surg. 1987;93:539-45.

2. Rowlatt UF, Rimoldi HJA, Lev M. The quantitative anatomy of the normal child's heart. Pediatr Clin North Am. 1963;10:499-588.

3. McCrindle BW, Tchervenkov CI, Konstantinov IE, Williams WG, Neirotti RA, Jacobs ML, et al. Risk factors associated with mortality and interventions in 472 neonates with interrupted aortic arch: a Congenital Heart Surgeons Society study. J Thorac Cardiovasc Surg. 2005; 129:343-50

4. Ohye RG, Kagisaki K, Lee LA, Mosca RS, Goldberg CS, Bove EL. Biventricular repair for aortic atresia or hypoplasia and ventricular septal defect. J Thorac Cardiovasc Surg. 1999;118:648-53.

5. Black MD, Smallhorn JF, Freedom RM. Aortic atresia with a ventricular septal defect: modified single-stage neonatal biventricular repair. Ann Thorac Surg. 1999;67:751-5.

\title{
A new cannula for innominate artery cannulation
}

\author{
Paolo Stassano, MD, Antonino Musumeci, MD, Gabriele lannelli, MD, Giuseppe D'Alise, CCCP, and \\ Michele Mottola, MD, Naples, Italy
}

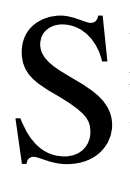

urgical treatment of Stanford type A aortic dissection remains a difficult challenge. Among of the first problems that the surgeon faces are the site and the type of arterial cannulation, which are of paramount importance in having a good arterial flow, ensuring a good body perfusion without damaging organs, not extending the dissection, speeding up surgical correction, and ensuring antegrade post-correction flow.

We developed a modified right-angled cannula that allows cannulation of the innominate artery (IA) and uniform perfusion throughout the procedure.

\section{Methods}

A preoperative computed axial tomography scan clearly identifies the extent of the dissection and the vessels involved. The right radial artery is cannulated for arterial pressure recording. A right groin incision is performed, and the femoral vessels are exposed but not cannulated. Median sternotomy is performed, and the IA is isolated. After systemic heparinization, the IA is cannulated with our modified cannula. We use a $20 \mathrm{~F}$ to $22 \mathrm{~F}$ cannula with a supplemental hole in the elbow. The width of this hole is similar to the distal cannula's hole (Figure 1). This arterial cannula is connected to the arterial line of the extracorporeal circuit. The venous return to the extracorporeal circuit is ensured by a single venous cannula in the right atrium.

Cardiopulmonary bypass is started with the arterial inflow ensured by the cannula in the IA.

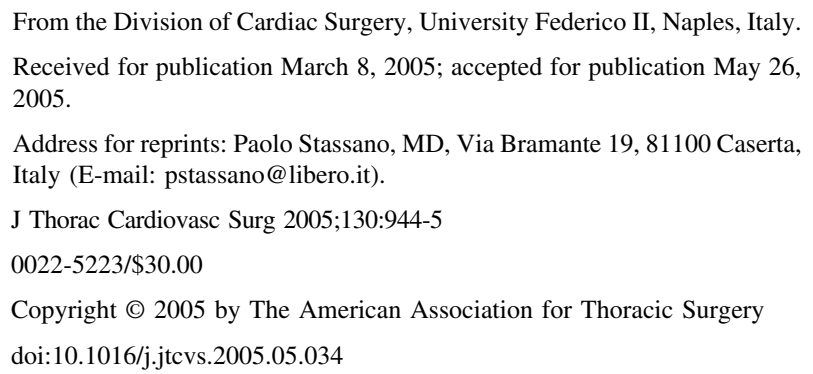

The aorta is clamped, and a longitudinal incision is made in the ascending aorta. The tear is usually seen, frequently extending into the aortic arch. Myocardial protection is achieved with intermittent blood cardioplegia delivered directly into the coronary arteries. The diseased aorta is excised, and moderate patient cooling is started. Meanwhile, the proximal aortic stump is repaired with BioGlue (CryoLife, Kennesaw, Ga), the aortic valve is resuspended, and a graft is sutured to the proximal aortic stump. At $24^{\circ} \mathrm{C}$ esophageal temperature, the IA is clamped proximally to the cannulation site (Figure 2). Cerebral perfusion through the cannula in the IA is continued at $10 \mathrm{~mL} \cdot \mathrm{kg} \cdot \min$ and adjusted to maintain a mixed venous oxygen saturation of $85 \%$ to $95 \%$, while body perfusion is momentarily stopped. The left carotid and left subclavian arteries are also clamped or occluded. Then the aorta is declamped, resection and repair of the distal ascending aorta and/or hemiarch are completed, and distal anastomosis of the tube graft is completed. With the patient in the Trendelenburg position, the flow through the IA cannula is increased gradually as the clamps on the brachiocephalic vessels are released and body perfusion is restarted exclusively through this cannula. The patient is rewarmed to $37^{\circ} \mathrm{C}$ esophageal temperature, and surgery is completed.

\section{Comment}

In type A aortic dissection many arterial cannulation sites have been proposed with the aim of providing a good and homogeneous perfusion and safe central nervous system protection.

The IA is usually spared from the dissecting lesion, easily prepared, and of sufficient caliber to allow antegrade and retrograde flow. ${ }^{1}$ The perfusion achieved with its cannulation is good and allows an excellent correction of the distal portion of the ascending aorta and/or the arch, without the cumbersome presence of the clamp. The surgical procedure can be accomplished without undue haste, because antegrade cerebral perfusion is never interrupted and the central nervous system protection is provided with a blood flow of $10 \mathrm{~mL} \cdot \mathrm{kg} \cdot \mathrm{min}$. Furthermore, deep hypothermia is not used, thus avoiding its potential hazards.

Concerns may be raised about the adequacy of cerebral perfusion, which relies on unilateral cerebral perfusion, but extensive anastomoses exist between the vertebral and the ca- 